\title{
Knowledge and attitude of youth towards contact lenses in Karachi, Pakistan
}

\author{
Rafia Irfan', Roha Saeed Memon', Mahnoor Yousif Shaikh1', Ilma Khalid', Nimra Shakeel', Eleze Tariq ${ }^{2}$ \\ 1 Dow University of Health Sciences, Karachi, Sindh, Pakistan, ${ }^{2}$ Aga Khan Hospital Karachi, Sindh, Pakistan \\ Keywords: global health \\ https://doi.org/10.29392/joghr.3.e2019042
}

\section{Journal of Global Health Reports}

Vol. 3, 2019

\begin{abstract}
Background
Surveys have shown that contact lenses are common optical correction devices used worldwide and they are particularly popular among the youth. However, not much is known about the knowledge and attitude of youth towards contact lenses in Pakistan. This study targeted to assess the approach of youth in Karachi towards contact lenses and whether the differences noted in approach was simulated by gender.
\end{abstract}

\section{Methods}

We conducted a cross-sectional survey in September 2018, using convenience sampling and interviewed 400 individuals, aged 16-25, to assess their knowledge, attitude, and practices towards contact lenses. The respondents were asked about their knowledge and its source. Attitudes were assessed by asking participants opinions, while practice question mainly focused on the recent (up to 1 month) use of contact lenses and their experience. Chi-square test was applied to cross-tabulate gender with knowledge, attitude, and practices of youth towards contact lenses.

\section{Results}

The majority of the participants knew what contact lenses were $(n=388,97.0 \%)$ but did not know about the different materials of contact lenses available $(n=280,70.0 \%)$ and had learnt about them from their family $(n=188,47.0 \%)$. More than half of them $(n=211$, $52.8 \%$ ) believed that the reason for contact lens use was to enhance physical appearance. An overwhelming majority stated that contact lenses should be cleaned daily ( $n=211$, $52.8 \%$ ) and they would never recommend contact lenses use in shower, pools or beaches $(n=395,98.8 \%)$. Only about one-third $(n=126,31.5 \%)$ of the participants stated that they wear contact lenses and a vast majority of them said that they wear them only on special occasions ( $n=88,69.8 \%)$. Statistically significant differences were found with females knowing more about the possible side effects of using contact lenses $(P=0.001)$ and being a current contact lens user $(P<0.001)$.

\section{Conclusions}

This study shows that a lot of young adults residing in Karachi have significant knowledge about contact lens, however, their knowledge about its proper care and storage seems poor. Moreover, they seem to be non-compliant to the standard hygiene recommended by eye practitioners. Males and females had substantial differences in their outlook towards contact lenses and their use mainly due to the fact that females are more concerned about their physical appearance.

An estimated $80 \%$ of blindness worldwide is avoidable. ${ }^{1}$ Uncorrected refractive error is one of the major causes of avoidable visual disability, due to which there is an enormous need globally for devices and treatments that can be used for its correction. One of the correction devices is contact lenses, which are thin optical devices placed in direct contact with the surface of the eye. They lie immediately above the cornea, adhering to the film of tears due to surface tension. ${ }^{2,3}$ Contact lenses are of several types, each type designed to meet a different purpose. They are classified by their primary function (corrective, multifocal, monovision contact lenses) and material (rigid, soft and hybrid contact lenses). Contact lens usage ranges from therapeutic to cosmetic to sports. Contact lenses provide better peripheral vision and also slows the progression of myopia in individuals as compared to spectacles. ${ }^{4}$ Since their use is believed to be associated with enhancing the aesthetics of 
the wearer, its use has been quite popular among teenagers and young adolescents in the past years.

In 2004, it was estimated that 125 million people worldwide use contact lenses. ${ }^{5}$ Whereas in the US alone, an estimated 40.9 million adults wear contact lenses. ${ }^{6}$ These constitute $16.7 \%$ of the total US adult population, with most of them being younger, females and more educated compared with those who don't wear contact lenses. ${ }^{7}$ As of 2010, the average estimated age of contact lens wearers was 31 years old globally, and two-thirds of all the wearers were females. ${ }^{8}$ Increased prevalence among young adolescents is mainly due to the increased variety in terms of lens type, material, shades, easy over-the-counter availability and cheaper costs as compared to the past. In spite of undergoing constant advancement contact lenses are associated with complications and side effects which can range in severity from simple allergic reactions to more complex corneal ulcers. ${ }^{9}$ According to a survey, mishandling and improper hygiene techniques play an important role in giving rise to such complications. Another study shows that despite the complications due to contact lenses, $87 \%$ of people claimed to prefer contact lenses for the cosmetic purpose. 10 Much is known about the contact lens use in children. ${ }^{11-14}$ Although substantial amounts of studies have been conducted regarding the topic of contact lenses and their use by youth, there is a lack of data in different parts of the world, such as Pakistan, regarding the perceptions and knowledge of youth towards contact lenses.

This study focuses primarily, on youth's knowledge and attitude towards contact lenses in Karachi, Pakistan, while having a secondary focus on youth practices regarding the use of contact lenses and its storage in order to reduce contact lens-associated complications. Since contact lenses are very popular amongst the youth they should have profound knowledge regarding its risk, benefits and proper hygiene techniques as lack of knowledge can cause adverse effects like epithelial changes, papillary conjunctivitis, allergy and ulcerative keratitis. Youth should be well aware of the importance of discontinuing the use of contact lenses and consult an eye specialist when acute ocular irritation develops in order to prevent irreversible damage to eyes.

\section{METHODS}

In September 2018, we conducted a 4-week cross-sectional study to assess the knowledge and attitude of youth in Karachi towards contact lenses. The study was approved by the Institutional Review Board of Dow University of Health Sciences (DUHS). The sample size was calculated using a confidence level of $95 \%$ and a frequency of outcome factor of $50 \%$. The computed sample size was 384 . Keeping our margin, we took 400 samples and interviewed individuals using convenience sampling. Any individuals aged 16-25 years were selected. Only 2 restrictions were placed that was age and city of residence i.e. Karachi. Other than that no socioeconomic limitations like income and level of education were placed. Initially, the questionnaires were designed in the English Language. They were also translated in the native language i.e. Urdu using Google Translate for people who were unable to understand English completely. The questions were in simple and easy language, in order to make it easily understandable for the participants. Written consent was taken from the participants after thoroughly explaining the purpose of the study to them. Every participant was given the right to withdraw from the study at any point they wished to do so. Two researchers were allotted the duty of conducting the survey. One researcher was responsible for conducting the survey in English, while the other conducted it in Urdu. Both of them provided a clear explanation of the questions and ensured that the contents and meaning of the questions were preserved while surveying in either of the two languages. This was done to minimize instruction and interviewer bias. Response bias was minimized by ensuring anonymity and in order to reduce recall bias, practice questions focused mainly on recent practices, up to one month.

The questionnaire comprised of 21 questions and was divided into three sections, namely knowledge, attitude, and practice. In the knowledge section information was collected about whether the respondents knew about contact lenses, their types, their side effects and the source of this knowledge. In the attitude section, respondents were asked about their perspective on the reasons for the usage of contact lenses and appropriate hygiene behavior. Regarding practices, the use of contact lenses and their side effects were inquired.

Data was entered and analyzed using SPSS version 23.0. Frequencies and percentages were computed for categorical responses. Chi-square test was applied to cross-tabulate gender with knowledge, attitudes, and practices of youth towards contact lenses. $P$-value $<0.05$ was considered as statistically significant.

\section{RESULTS}

Out of 400 individuals who completed the questionnaire, the majority $(n=274,68.5 \%)$ were females. The mean age of the individuals was $20.3 \pm 1.7$ years, with more than onefourth ( $\mathrm{n}=109,27.3 \%)$ being 21 years old.

An overwhelming majority of individuals ( $n=388,97.0 \%)$ knew what contact lenses were and almost half of these individuals ( $\mathrm{n}=188,47.0 \%)$ learnt about contact lenses from their family while about one-fifth of them $(n=75,18.8 \%)$ found out through media. We also found that almost threefourth of the individuals $(n=280,70.0 \%)$ were not aware of the different materials of contact lenses available, however, a vast majority of these individuals were aware of the possible side effects of wearing contact lenses $(n=364$, 91.0\%). Among all the people who were aware of the possible side effects, about one-third were aware of eye infections $(n=311,36.2 \%)$ as well as itching and redness $(n=305$, $35.5 \%)$.

More than half of the individuals $(n=211,52.8 \%)$ said that people wear contact lenses to enhance their physical appearance while more than one-fifth of the individuals $(n=86,21.5 \%)$ believed that people wear contact lenses because they find them more convenient. A greater portion of the participants $(n=211,52.8 \%)$ were conscious about the fact that contact lenses should be cleaned daily, while a small portion ( $n=147,36.8 \%)$ believed that it should be done on weekly basis. We also discovered that almost three-fifths of the participants $(n=234,58.5 \%)$ considered laziness as 
one of the most important reasons that hold people back from cleaning their contact lenses. Almost all the individuals $(n=395,98.8 \%)$ refused to recommend contact lenses in shower, pool or beaches.

It was found that only about one-third of the participants $(\mathrm{n}=126,31.5 \%)$ wear contact lenses and out of those, majority of the individuals $(n=88,69.8 \%)$ wear them only on special occasions. Furthermore, nearly a quarter of the participants ( $n=82,20.5 \%)$ stated that they wear contact lenses for five consecutive hours and have never slept with contact lenses on ( $\mathrm{n}=95,23.8 \%)$. Additionally, only a small portion of the participants $(n=54,13.5 \%)$ admitted that have had experienced side effects because of contact lens use. In addition to this about one-third ( $\mathrm{n}=141,35.5 \%)$ of the individuals were not sure of whether they will try contact lenses in future or not and said that they would recommend $(n=123$, $30.8 \%$ ) and promote contact lens use.

The results of various questions, as percentages of the whole sample population, when compared with the gender of the individual have been presented in Table 1.

Females significantly came to know about contact lenses from their family $(P=0.014)$ and were aware of the possible side effects of wearing contact lenses $(P=0.001)$. Whereas significantly more males chose the option 'I don't know about the possible side effects of wearing contact lenses' $(P=0.001)$. Furthermore, statistically significant differences were also found when comparing gender with if the individuals wear contact lenses $(P=0.025)$ or not and if they would promote or recommend $(P<0.001)$ contact lenses to others. More females answered yes to the aforementioned questions.

\section{DISCUSSION}

Our principal finding was that almost all the participants did have substantial knowledge about contact lenses and showed a positive attitude towards its use.

In contrast, a similar study was conducted in Ghana where it was seen that a very small percentage of the sample population was aware of the use of contact lenses as vision correcting agents and even fewer people had a history of contact lens use. However, it was seen in this study that out of those who knew about contact lenses the majority of them were young adults. ${ }^{15}$ This was consistent with our study and this consistency is perhaps due to the fact that young adults are more self-conscious and they often feel more accepted without spectacles. Contact lenses may also help to boost their self-esteem and may offer an extra benefit to those youth who are active in sports. This is analogous to a previous study which concluded that young adult tends to wear contact lenses in order to enhance their physical appearance. ${ }^{16}$ This compounded by the fact that lenses and spectacles both allow for the same effect in terms of vision correction makes lenses an easier choice for most youth.

Previous studies have shown that the major sources of information to people were the internet, magazines, and television. ${ }^{17}$ We however discovered in our study that family was the major source of information to these young adults and this is perhaps due to the immense importance of family values in Pakistani culture, where family plays a major role in influencing an individual's lifestyle, decisions, and social activities.

Furthermore, we found statistical variations in the knowledge, attitudes, and practices among male and females with females having a more acquiescent stance regarding contact lenses with more of them being current user as shown by previous studies. ${ }^{18-20}$ This is probably due to females being more diligent about their health and appearance.

We also found that the majority of the participants were unaware of the different materials of contact lenses available. This could be due to lack of advertising and branding of contact lenses in Pakistan. The knowledge regarding the possible side effects of the participants in our study seemed sound. In a recent study conducted in 2011, people were found to be non-compliant with the proper hygiene and storage method of contact lens even when they were aware of the possible risks associated with it. This is reflected by our study, which shows that young adults using contact lens specifically in Karachi also consider themselves non-compliant although they are aware of the possible side effects and this is, as they pointed, due to lack of time or sheer laziness. ${ }^{21}$ In the same study, more than half of the participants were reported to use contact lens while swimming and of those, 50\% knew the possible infections that this activity could cause yet they still kept at it. However, in our study, almost all participants refused to advice people to wear contact lenses while in contact with water, whether it is swimming pools or beaches. Furthermore, most of the individuals in our study wore contact lenses only on special occasions and did not recommend its daily use. This is perhaps due to the daily cleaning and care is considered tedious by youth. According to a study conducted to determine the possible risk of wearing contact lens for an extended time period and their overnight use, contact lens worn during sleeping cause high rate of corneal infections due to oxygen deprivation to the inner surface of the eye. ${ }^{22}$ Regardless of this fact, only one-fourth of the people in our study admitted having never worn lens during sleep. This could be due to the fact that participants feel lazy to remove the lens or simply because they don't consider it important.

Our study showed that the participants had sufficient knowledge regarding contact lenses. However, there was a lack of compliance and the improper handling of contact lenses. This could result in contamination leading to infection which could be harmful to the eyes. To reduce the risk of contact lens complications, good habits of contact lens wearers in regard to lens care are of utmost importance. Therefore, better training of contact lens providers is essential, and every contact lens wearer, even educated young people, should have a better understanding of the importance of good lens care practices in order to prevent ocular complications. This can be achieved by guiding lens providers to educate their customers about the different types and risks of contact lenses to ensure standardized practice and sale thereby minimizing the risk of complications. In addition, majority of the participants were uninformed about the different types of contact lenses hence contact lens providers should first thoroughly educate the patients regarding contact lenses before suggesting them as vision correction tools in order to reduce the prevalence 
Table 1. Knowledge, attitudes and practices compared with gender

\begin{tabular}{|c|c|c|c|c|}
\hline \multirow{2}{*}{ Questions } & \multirow{2}{*}{ Response options } & \multicolumn{2}{|c|}{ Gender } & \multirow{2}{*}{$P$-value } \\
\hline & & Male & Female & \\
\hline \multirow[t]{2}{*}{ Do you know about contact lenses } & Yes & $122(30.5 \%)$ & $266(66.5 \%)$ & \multirow{2}{*}{0.890} \\
\hline & No & $4(1.0 \%)$ & $8(2.0 \%)$ & \\
\hline \multirow[t]{6}{*}{ If yes, then how did you came to know about them? } & Friends & $23(5.75 \%)$ & 75(18.75\%) & \multirow{6}{*}{0.014} \\
\hline & Media & $27(6.75 \%)$ & $48(12.0 \%)$ & \\
\hline & Family & $67(16.75 \%)$ & $121(30.25 \%)$ & \\
\hline & Optician & $2(0.5 \%)$ & $21(5.25 \%)$ & \\
\hline & I don't know about contact lenses & $4(1 \%)$ & $8(2 \%)$ & \\
\hline & Other & $3(0.75 \%)$ & $1(0.25 \%)$ & \\
\hline \multirow[t]{2}{*}{ Are you aware of the different types of contact lenses available? } & Yes & $41(10.25 \%)$ & 79(19.75\%) & \multirow{2}{*}{0.452} \\
\hline & No & $85(21.25 \%)$ & 195(48.75\%) & \\
\hline \multirow[t]{2}{*}{ Are you aware of the possible side effects of wearing contact lenses? } & Yes & $106(26.5 \%)$ & $258(64.5 \%)$ & \multirow{2}{*}{0.001} \\
\hline & No & $20(5.0 \%)$ & $16(4.0 \%)$ & \\
\hline \multirow[t]{7}{*}{ Why do you think people wear contact lenses? } & To enhance their physical appearance & $76(19.0 \%)$ & $135(33.75 \%)$ & \multirow{7}{*}{0.108} \\
\hline & Contact lenses make them see well & $10(2.5 \%)$ & $25(6.25 \%)$ & \\
\hline & $\begin{array}{l}\text { Spectacles have a limiting effect on their } \\
\text { life }\end{array}$ & $11(2.75 \%)$ & $30(7.5 \%)$ & \\
\hline & $\begin{array}{l}\text { They find contact lenses more } \\
\text { convenient }\end{array}$ & $24(6.0 \%)$ & $62(15.5 \%)$ & \\
\hline & It boosts their self-esteem & $1(0.25 \%)$ & $17(4.25 \%)$ & \\
\hline & Peer pressure & $2(0.5 \%)$ & $4(1.0 \%)$ & \\
\hline & Other & $2(0.5 \%)$ & $1(0.25 \%)$ & \\
\hline \multirow{6}{*}{$\begin{array}{l}\text { What do you think is the most important reason that holds people back from cleaning their } \\
\text { contact lenses? }\end{array}$} & They find it tedious & $14(3.5 \%)$ & $27(6.75 \%)$ & \multirow{6}{*}{0.707} \\
\hline & They find it time-consuming & $14(3.5 \%)$ & $26(6.50 \%)$ & \\
\hline & They are too lazy to do it & $76(19.0 \%)$ & $141(39.5 \%)$ & \\
\hline & $\begin{array}{l}\text { They don't feel its imp to clean contact } \\
\text { lenses }\end{array}$ & $16(4.0 \%)$ & $51(12.75 \%)$ & \\
\hline & They find it very costly & $4(1.0 \%)$ & $10(2.50 \%)$ & \\
\hline & Other & $2(0.5 \%)$ & $2(0.5 \%)$ & \\
\hline Do you wear contact lenses? & Yes & $30(7.50 \%)$ & $96(24.0 \%)$ & 0.025 \\
\hline
\end{tabular}




\section{How often do you wear contact lenses?}

Do you intend to wear contact lenses in future?

Will you recommend people to wear contact lenses?
No

Daily

Weekly

Monthly

Only on special occasions

I don't wear contact lenses

Yes

No

Maybe

I already wear contact lenses

Yes

No

Maybe
96(24.0\%)

$3(0.75 \%)$

$2(0.5 \%)$

$0(0.00 \%)$

$24(6.0 \%)$

$30(7.5 \%)$

$5(1.25 \%)$

44(11.0\%)

47(11.75\%)

$30(7.5 \%)$

$34(8.5 \%)$

$31(7.75 \%)$

61(15.25\%)
178(44.5\%)

$13(3.25 \%)$

11(2.75\%)

9(2.25\%)

63(15.75\%)

96(24.0\%)

$33(8.25 \%)$

$51(12.75 \%)$

94(23.5\%)

96(24.0\%)

$89(22.25 \%)$

67(16.75\%)

$118(29.5 \%)$
0.053

0.000

0.496 
of contact lens-associated complications. Furthermore, all contact lens users should be encouraged to pay a visit to the ophthalmologist as soon as they detect the first signs of irritation so as to ensure early and complete recovery.

This study is limited in the sense that it only assesses whether teenagers in Karachi know about contact lenses, further studies should be carried out in other areas of Pakistan which may cover small rural areas and different demographics. There should also be studies comparing the effect of lenses and spectacles on vision correction. Since most of the teenagers claimed that contact lenses should be cleaned daily there should also be studies focusing on the compliance of the lens care routine. The study can also be conducted with different age groups and their responses can be compared to see if there are any changes in knowledge and attitude. Since only youth in Karachi were particularly targeted in this survey, different standpoints were not gathered since the participants did not include youth from different areas and socioeconomic backgrounds. Other than that the sample was not randomized hence there was a risk of selection bias. Additionally, there was a disproportionate ratio between boys and girls as more than half of the participants in our study were females, therefore, it was not representative of the entire youth population of Karachi.

\section{ACKNOWLEDGEMENTS}

This study was approved by the Institutional Review Board of Dow University of Health Sciences (DUHS).

\section{FUNDING}

None.

\section{COMPETING INTERESTS}

The authors have completed the Unified Competing Interest form at http://www.icmje.org/coi disclosure.pdf (available on request from the corresponding author) and declare no conflict of interest.

\section{CORRESPONDENCE TO:}

Rafia Irfan

3rd year student of MBBS

\#403, 4th floor, K-block, Creek vista apartments

DHA phase 8

Karachi, Sindh, Pakistan

rafiairfan96@live.com 


\section{REFERENCES}

1. Thylefors B. A global initiative for the elimination of avoidable blindness. Am J Ophthalmol. 1998;125(1):90-93. doi:10.1016/s0002-9394(99)8023 9-6

2. Holden BA. Uncorrected refractive error: the major and most easily avoidable cause of vision loss. Community Eye Health. 2007;20:37-39.

3. Moreddu R, Vigolo D, Yetisen AK. Contact Lens Technology: From Fundamentals to Applications. Adv Healthcare Mater. 2019;8(15):1900368. doi:10.1002/ad hm.201900368

4. Backhouse S, Fox S, Ibrahim B, Phillips JR. Peripheral refraction in myopia corrected with spectacles versus contact lenses. Ophthalmic Physiol Opt. 2012;32(4):294-303. doi:10.1111/j.1475-1313.20 12.00912.X

5. Farandos NM, Yetisen AK, Monteiro MJ, Lowe CR, Yun SH. Contact lens sensors in ocular diagnostics. Adv Healthcare Mater. 2014;4(6):792-810. doi:10.100 2/adhm.201400504

6. Cope JR, Collier SA, Rao MM, et al. Contact lens wearer demographics and risk behaviors for contact lens-related eye infections-United States, 2014. MMWR Morb Mortal Wkly Rep. 2015;64(32):865-870. ㅁ oi:10.15585/mmwr.mm6432a2

7. Nichols JJ. Contact Lenses 2015; Strong growth in some segments and a few surprises highlight a year of modest gains overall. Contact Lens Spectr. 2016;31(18-23):55.

8. Morgan PB, Efron N, Helland M, et al. Demographics of international contact lens prescribing. Cont Lens Anterior Eye. 2010;33(1):27-29. doi:10.1016/j.clae.2009.09.006

9. Morgan PB, Efron N, Hill EA, Raynor MK, Whiting $\mathrm{MA}$, Tullo $\mathrm{AB}$. Incidence of keratitis of varying severity among contact lens wearers. Br J Ophthalmol. 2005;89(4):430-436. doi:10.1136/bjo.2004.052688

10. Janti S, Charanya C, Raja AM, Matheen A, Pandurangan R. Knowledge, Attitude, and Practice of Contact Lens Users among Medical College Students in Tamil Nadu. Int J Sci Stud. 2014;2:20-23.

11. Dias L, Manny RE, Weissberg E, Fern KD. Myopia, contact lens use and self-esteem. Ophthalmic Physiol Opt. 2013;33(5):573-580. doi:10.1111/opo.12080
12. Kemper AR, Bruckman D, Freed GL. Prevalence and distribution of corrective lenses among schoolage children. Optom Vis Sci. 2004;81(1):7-10. doi:10.1 097/00006324-200401000-00003

13. Walline JJ, Long S, Zadnik K. Daily disposable contact lens wear in myopic children. Optom Vis Sci. 2004;81(4):255-259. doi:10.1097/00006324-20040400 0-00011

14. Walline JJ, Jones LA, Sinnott L, et al. Randomized trial of the effect of contact lens wear on selfperception in children. Optom Vis Sci. 2009;86(3):222-232. doi:10.1097/opx.0b013e3181971 $\underline{985}$

15. Abokyi S, Manuh G, Otchere H, Ilechie A. Knowledge, usage and barriers associated with contact lens wear in Ghana. Cont Lens Anterior Eye. 2017;40(5):329-334. doi:10.1016/j.clae.2017.05.006

16. Plowright AJ, Maldonado-Codina C, Howarth GF, Kern J, Morgan PB. Daily disposable contact lenses versus spectacles in teenagers. Optom Vis Sci. 2015;92(1):44-52. doi:10.1097/opx.000000000000045 $\underline{4}$

17. Riley C, Chalmers RL. Survey of contact lenswearing habits and attitudes toward methods of refractive correction: 2002 versus 2004. Optom Vis Sci. 2005;82(6):555-561. doi:10.1097/01.opx.0000167 $\underline{104.81142 .40}$

18. Unnikrishnan B, Hussain S. Pattern of use of contact lens among college students: a crosssectional study in coastal Karnataka. Indian J Ophthalmol. 2009;57(6):467. doi:10.4103/0301-4738.5 $\underline{7159}$

19. Lee YC, Lim CW, Sam SM, Koh D. The prevalence and pattern of contact lens use in a Singapore community. CLAO J. 2000;26:21-25.

20. Riley C, Chalmers RL. Survey of contact lens wearing habits and attitudes towards methods of refractive correction: 2002 versus 2004. Optom Vis Sci. 2005;82(6):555-561. doi:10.1097/01.opx.0000167 $\underline{104.81142 .40}$

21. Bui TH, Cavanagh HD, Robertson DM. Patient compliance during contact lens wear: perceptions, awareness, and behavior. Eye Contact Lens. 2010;36(6):334-339. doi:10.1097/icl.0b013e3181f579f 7 
22. Goodlaw E. Risk of infection from sleeping with contact lenses on: causes of risk. Optom Vis Sci. 1996;73(3):156-158. doi:10.1097/00006324-19960300 $\underline{0-00005}$ 